\title{
La evolución del e-learning: del aula virtual a la red
}

\section{The evolution of e-learning: from virtual classroom to the network}

\author{
Begoña Gros Salvat \\ Universidad de Barcelona (España)
}

\section{Resumen}

El objetivo fundamental de este artículo es analizar la evolución y los retos del e-learning haciendo especial énfasis en la necesidad de pasar de un espacio cerrado -el aula virtuala incorporar herramientas adaptativas que garanticen un uso mucho más personalizado. En el artículo se describe la evolución del e-learning desde el punto de vista pedagógico y tecnológico y se muestra como los espacios formativos se han diversificado más allá del aula virtual. El e-learning está incorporando diseños pedagógicos más abiertos en que diferentes personas pueden compartir contenidos, actividades y experiencias en situaciones formales e informales. Numerosas investigaciones muestran como en los entornos de aprendizaje en línea tienden a fracasar con los estudiantes con menos capacidad de autorregulación. Por el contrario, los estudiantes más exitosos muestran una mayor eficacia y eficiencia en los procesos de autorregulación. Las investigaciones sobre el papel de los estudiantes en los entornos virtuales de aprendizaje destacan la importancia de los procesos de autorregulación en el éxito de los resultados. Las tecnologías emergentes tales como el uso de sistemas adaptativos, los agentes inteligentes y el uso de las analíticas de aprendizaje pueden facilitar los procesos de adaptación y autorregulación ya que permiten ejercer una doble función: proporcionar información en tiempo real a los aprendices y facilitar estrategias de andamiaje durante el proceso de aprendizaje.

Palabras clave: educación a distancia; educación abierta; aprendizaje abierto; e-learning; blended learning.

\begin{abstract}
The main goal of this article is to analyse the evolution and challenges of e-learning, with special emphasis on the need to move from a closed space-the virtual classroom-to incorporate adaptive tools that guarantee a much more personalized use. The article describes the evolution of e-learning from a pedagogical and technological point of view and shows how the training spaces have diversified beyond the virtual classroom. E-learning is incorporating more open pedagogical designs in which different people can share content, activities and experiences in formal and informal situations. Research in this field shows that students with less selfregulation capabilities tend to fail. On the contrary, the most successful students show greater efficiency and efficiency in self-regulated learning. Research on the role of students in virtual
\end{abstract}


learning environments highlights the importance of self-regulation processes in the success of the results. Emerging technologies such as the use of adaptive systems, intelligent agents and the learning analytics can facilitate the processes of adaptation and self-regulation. The adoption of these technology can provide real-time information to learners and to facilitate strategies for scaffolding during the learning process.

Keywords: distance education; open education; open learning; e-learning; blearning.

En los últimos años la educación en línea se ha expandido y consolidado ampliamente en todos los niveles educativos y en situaciones formales y no formales de aprendizaje. Son muchas las causas que están impulsando el auge de este tipo de formación tales como una mayor integración de las tecnologías digitales en la vida diaria y en la formación, la flexibilidad de acceso, la individualización, el aprendizaje activo, etc.

El e-learning nació en los años noventa como una modalidad de formación asociada a la educación a distancia y al uso de Internet en la educación superior y la formación empresarial. Conviene destacar que existe una diferencia importante entre los modelos tradicionales de educación a distancia y el e-learning. La educación a distancia puede o no utilizar tecnología, pero lo más importante es garantizar el estudio independiente sin necesidad de que haya una intervención continua del docente. En el caso del e-learning, se comparte la no presencialidad del modelo, pero el énfasis se produce en la utilización de Internet como sistema de acceso a los contenidos y a las actividades de la formación. Además, la interacción y la comunicación son una parte fundamental en este tipo de formación.

La evolución del e-learning está sujeta a las transformaciones tecnológicas y a los cambios relacionados con los espacios y tiempos para la formación y el aprendizaje. Inicialmente, el e-learning estaba vinculado al uso de los ordenadores personales y actualmente está sostenido por los sistemas distribuidos que han favorecido las redes de aprendizaje y el acceso a las plataformas desde cualquier dispositivo. Así, el uso de dispositivos móviles se ha integrado en los diseños educativos y las actividades de aprendizaje en línea.

El objetivo principal de este artículo es analizar la evolución del e-learning y los retos que supone la mejora de este tipo de modalidad de aprendizaje. El artículo comienza con una descripción de los avances del e-learning desde el punto de vista pedagógico y tecnológico. En el segundo apartado, abordamos la diversidad de espacios formativos y la combinación de los espacios presenciales y virtuales. A continuación, examinamos los elementos diferenciales que caracterizan la formación en línea y el papel del aprendiz en dichos entornos. Y, finalmente, analizamos la contribución de las tecnologías para mejorar la adaptación y personalización del e-learning. 


\section{LA EVOLUCIÓN TECNO-PEDAGÓGICA DEL E-LEARNING}

No pretendemos realizar un análisis exhaustivo de la historia y evolución del e-learning ${ }^{1}$ (Gros y García Peñalvo, 2016) pero sí que consideramos importante señalar algunos hitos relevantes desde el punto de vista pedagógico y tecnológico. Downes (2012) describe la evolución del e-learning a partir de una serie de generaciones que no necesariamente son excluyentes, sino que han ido conviviendo a lo largo del tiempo. En primer lugar, señala la existencia de una generación cero caracterizada por el diseño y la publicación de los recursos multimedia en línea. En este primer momento, lo más importante es usar los ordenadores para transmitir contenidos instructivos y realizar actividades basadas en pruebas y cuestionarios evaluativos. La generación uno del e-learning se inicia a partir de Internet y el uso del correo electrónico que facilita la comunicación virtual. La segunda generación tiene lugar a principios de los noventa y se caracteriza por la aplicación de los juegos de ordenador para el aprendizaje en línea. En la tercera generación, el desarrollo de los gestores de aprendizaje (LMS) permite conectar los contenidos de la generación cero con las plataformas. De hecho, para muchos autores esta fase es muy relevante ya que el e-learning se materializa en el aula virtual. Por ejemplo, Area y Adell (2009, p. 2) señalan como el e-learning "tiene lugar totalmente o en parte a través de una especie de aula o entorno virtual en el cual tiene lugar la interacción profesor-alumnos así como las actividades de los estudiantes con los materiales de aprendizaje”. La generación cuatro se fundamenta en el uso de la Web 2.o. Una de las características más significativas de esta fase es la interacción social entre los alumnos, cambiando la naturaleza de la red subyacente donde los nodos son ahora personas en lugar de ordenadores. Esta orientación social también se ve impulsada gracias al uso de los dispositivos móviles. La generación cinco se caracteriza por la computación en la nube y el contenido abierto. Por último, la generación seis se caracteriza por los cursos abiertos masivos en línea (MOOCs).

Un elemento importante en la evolución descrita es que se van generando recursos y espacios cada vez más amplios y complejos en los que participan múltiples agentes. El aprendizaje ya no está encapsulado en un único lugar sino en un conjunto de nodos. Estos cambios los han descrito con mucho acierto García-Peñalvo y Seoane-Pardo (2015) quienes describen la evolución del e-learning a partir de tres generaciones. La primera generación se caracteriza por la aparición de las plataformas de aprendizaje en línea y la creación de las aulas y campus virtuales. Estos sistemas evolucionan y, en la segunda generación, las plataformas permiten apoyar la socialización, la movilidad y la interoperabilidad de datos. Esta segunda generación subraya el factor humano. La interacción entre compañeros y la comunicación entre profesores y estudiantes son elementos esenciales para un e-learning de calidad que pretende ir más allá de un simple proceso de publicación de contenidos. El desarrollo de la web 2.0, las tecnologías móviles y los recursos abiertos para el aprendizaje también son factores significativos de esta generación. La tercera y última generación se caracteriza 
porque el e-learning deja de estar asociado de forma exclusiva a las plataformas de aprendizaje en línea. Los LMS dejan de ser un componente único y monolítico y se convierten en un componente más de un ecosistema tecnológico orientado hacia el proceso de aprendizaje. En este sentido, García-Peñalvo y Seoane-Pardo (2015, p. 132) consideran el e-learning como "un proceso formativo, de naturaleza intencional o no intencional, orientado a la adquisición de una serie de competencias y destrezas en un contexto social, que se desarrolla en un ecosistema tecnológico en el que interactúan diferentes perfiles de usuarios que comparten contenidos, actividades y experiencias y que, en situaciones de aprendizaje formal, debe ser tutelado por actores docentes cuya actividad contribuya a garantizar la calidad de todos los factores involucrados".

En cada una de las etapas o fases mencionadas no solo hay un cambio o evolución tecnológica sino también, como señalan Siemens, Gašević, y Dawson (2015), hay un cambio en las relaciones que se establecen entre el control del aprendizaje, la propiedad de los datos, la integración del sistema en la institución y los enfoques de enseñanza-aprendizaje. En este sentido, se observa una clara tendencia a desarrollar herramientas tecnológicas menos estandarizadas y que se adapten con mayor facilidad a las necesidades de los estudiantes. Por ello, las nuevas versiones de los LMS incorporan cada vez más herramientas adaptativas para poder diseñar cursos individualizados con prestaciones para gestionar el curso, los usuarios, los grupos, etc., además de permitir la gestión de contenidos (García Peñalvo y Seoane-Pardo, 2015).

\section{VIRTUALIDAD Y PRESENCIALIDAD: LA DIVERSIDAD DE ESPACIOS}

Tal y como hemos señalado, la metáfora espacial del aula virtual generada a partir del uso de los LMS está perdiendo centralidad ya que no es el único componente de la educación en línea. Tampoco el aula virtual es utilizada únicamente en la formación virtual. En realidad, los espacios virtuales y presenciales se han ido combinando generándose diversos modelos formativos que pueden convivir en una misma institución. Poder aprender sin las limitaciones de espacio y desde diferentes dispositivos ya es una realidad que abre muchas posibilidades formativas. El uso de los dispositivos móviles resulta hoy incuestionable (Sung, Chang y Liu, 2016) ya que facilitan el aprendizaje interactivo, ubicuo e individualizado.

Actualmente conviven diversas modalidades formativas que utilizan los recursos digitales distribuidos en la red de forma abierta y/o en espacios virtuales cerrados dentro de las plataformas. De este modo, encontramos prácticas de formación presencial gestionada con apoyo del aula virtual. Este modelo está muy extendido en la enseñanza secundaria, bachillerato y también en las universidades. El aula virtual tiene aquí un carácter complementario a la actividad docente, es un espacio que sirve fundamentalmente para la gestión de los materiales y la organización del 
curso: colgar los apuntes y otros documentos de estudio, el programa, los horarios de tutorías, las calificaciones, etc. Generalmente no supone un planteamiento novedoso docente y mantiene el modelo comunicativo tradicional unidereccional: profesorestudiantes.

La formación semi-presencial o el aprendizaje híbrido (blended learning) es otra modalidad formativa que también está adoptando diversos formatos. En general, se caracteriza por combinar procesos de enseñanza-aprendizaje presenciales con otros que se desarrollan a distancia. En este caso, el aula virtual no solo es un recurso de apoyo a la enseñanza presencial, sino también un espacio en el que el docente genera y desarrolla acciones diversas para que sus alumnos aprendan. Este enfoque implica un buen diseño del tiempo y las actividades que se realizan en los diversos espacios para que haya coherencia entre ellas. Evidentemente, dentro de este modelo existen variantes o grados en función del peso temporal y del trabajo distribuido entre las situaciones presenciales y virtuales. Por ejemplo, el aula invertida (flipped classroom) que también es una modalidad de aprendizaje semipresencial, plantea un cambio del modelo tradicional docente. En este caso, se trata de que la adquisición de los conocimientos se realice con soporte tecnológico fuera del aula con el fin de utilizar el tiempo de clase presencial para el desarrollo de actividades que favorezcan el aprendizaje colaborativo y la participación activa. El aprendizaje comienza en forma individual para luego trasladarse al espacio de aprendizaje en grupo.

La formación a distancia no tiene un planteamiento pedagógico homogéneo ya que actualmente podemos encontrar cursos de e-learning "tradicionales" hasta MOOCs con diseños pedagógicos también diversos. En todos los casos son modalidades formativas que no requieren presencialidad física, se desarrollan en entornos exclusivamente virtuales y las acciones docentes, comunicativas y de evaluación tienen lugar en el marco del entono virtual. En el caso de los MOOCs, hay que tener presente que es una modalidad virtual que surge a partir de una re-orientación de los cursos educativos tradicionales de e-learning. Se necesitaba plantear nuevos diseños superando los grupos y las estructuras cerradas progresando hacia redes sociales abiertas de estudiantes con capacidad para auto dirigir su propio aprendizaje (Siemens, 2014). Independientemente de las diversas tipologías de MOOCs (Daniel, Vázquez y Gisbert, 2015), son cursos que ofrecen un currículum estructurado sobre un tema o tópico, pero de sus estudiantes se espera que sean autónomos y que manejen su propio aprendizaje realizando sus propias conexiones sociales y conceptuales para cubrir sus necesidades, elemento clave para el aprendizaje. Por lo tanto, nos referimos a una pedagogía basada en la construcción de conexiones, colaboraciones y el intercambio de recursos entre personas, construyendo una comunidad de estudiantes y aprovechando los flujos de información que hay en las redes (Siemens, 2005).

Esta combinación de espacios virtuales y presenciales no se da únicamente en la educación formal. La amplia penetración de la tecnología permite adquirir conocimientos en múltiples espacios no formalizados. Por este motivo, cada vez 
hay una mayor necesidad de generar sistemas que permitan vincular el aprendizaje obtenido en los entornos formales e informales. En este contexto, la evolución del elearning debe extender la formación más allá del aula virtual y sustentar las diferentes trayectorias personales. La mayoría de los LMS actuales son propiedad de compañías que controlan estrictamente sus plataformas, lo que dificulta la ampliación de sus funciones y la integración de recursos externos de manera que se adapten mejor a las necesidades. Por ello, es frecuente que el profesorado incorpore herramientas como Google Apps, Trello, Slack, Youtube, etc., a las que se accede fuera del LMS. En este sentido, el objetivo de la próxima generación de LMS es cambiar el enfoque y profundizar en las funciones que apoyen el aprendizaje y no solo las puramente administrativas. Actualmente, algunas organizaciones e instituciones están trabajando para visualizar y desarrollar la próxima generación de LMS (García Holgado y García Peñalvo, 2017) que pretende enfatizar una mayor personalización y acceso a una gama de herramientas digitales cada vez más amplia.

\section{LA EFICACIA DEL E-LEARNING}

Muchos de los estudios sobre la eficacia del e-learning (Noesgaard y Ørngreen, 2015) han empleado una metodología comparativa. Esto significa que la mayoría de las investigaciones se han centrado en conocer si es mejor aprender en un entorno presencial o en uno virtual sin destacar los beneficios y aspectos propios de cada modalidad formativa. En este sentido, Noesgaard y Ørngreen, (2015, p. 280) preguntan “¿Deben las diferentes modalidades tener las mismas medidas de rendimiento, o debemos considerar el aprendizaje electrónico como un proceso de aprendizaje único y por lo tanto utilizar diferentes definiciones de la eficacia?" Esta cuestión es importante porque la eficacia del e-learning puede ser analizado de diferentes maneras en función de los objetivos que se persigan lograr. Por ejemplo, podemos diseñar e-learning para mejorar la retención de los estudiantes, el desempeño laboral o la colaboración social y, obviamente, la medida para evaluar la efectividad será diferente en cada caso.

Siemens (2014) señala que las investigaciones coinciden en que uno de los requisitos más importantes en el aprendizaje en línea es el desarrollo de cursos bien diseñados que incorporen contenidos interactivos y atractivos, actividades que permitan la colaboración estructurada entre pares y plazos flexibles que permitan a los estudiantes organizar sus tiempos. A unas conclusiones similares llegan Darabi et al. (2013) quienes consideran que el mayor impacto en el rendimiento del estudiante se obtiene a través de diseños pedagógicamente 'ricos' que incluyen la presencia de los formadores, la interacción con los alumnos, la colaboración del estudiante y el seguimiento continuo.

Además de la calidad del diseño pedagógico, el éxito del aprendizaje en el e-learning depende en gran medida de la capacidad del estudiante para dirigir y gestionar su propio proceso de aprendizaje, estableciendo objetivos y estrategias 
adecuadas para alcanzar sus objetivos. Numerosas investigaciones (Rowe y Rafferty 2013; van Laer y Elen, 2016) muestran como los alumnos en los entornos de aprendizaje en línea tienden a fracasar por su falta de autonomía. Por el contrario, los estudiantes más exitosos muestran una mayor eficacia y eficiencia en los procesos de autorregulación (van Laer y Elen, 2016).

van Laer y Elen (2016) examinaron las publicaciones realizadas entre 1985 a 2015 sobre los atributos de los entornos semi-presenciales de aprendizaje que apoyan la autorregulación. En esencia, la investigación correlaciona positivamente el rendimiento del alumno con el uso de estrategias autorreguladas de aprendizaje a través del ensayo, la elaboración y las estrategias de organización, el tiempo y las estrategias de regulación del esfuerzo, así como las del pensamiento crítico y la búsqueda de información. Las estrategias de aprendizaje autorreguladas también están fuertemente relacionadas con la motivación. van Laer y Elen (2016) identificaron siete atributos que permiten apoyar los procesos de autorregulación: personalización, interacción, autenticidad, andamiaje, control del aprendiz, señales para la reflexión y para la calibración. Estos siete elementos deberían orientar según Van Laer y Elen (2016) - el diseño pedagógico de la formación virtual.

Tomando un enfoque ligeramente diferente, Kizilcec, Pérez-Sangustín y Maldonado (2017), intentaron encontrar la relación entre las estrategias del aprendizaje autorregulado más efectivas y el rendimiento académico. Para ello, realizaron una investigación sobre una muestra de 4.831 estudiantes de 6 MOOCs diferentes. $\mathrm{Al}$ analizar los registros de los estudiantes, el rendimiento del curso, las respuestas de la encuesta y las medidas en línea de las interacciones de contenido del curso, encontraron varias estrategias que afectaron el aprendizaje de los estudiantes. Los hallazgos destacaron que

el establecimiento de metas y la planificación estratégica predijeron el logro de los objetivos del curso, mientras que la búsqueda de ayuda se asoció con un menor logro de los objetivos. Los alumnos con mayores habilidades de autorregulación tuvieron más probabilidades de revisar los materiales del curso previamente estudiados, especialmente las evaluaciones del curso" (Kizilcec et al., 2017, p. 18).

Si la autonomía del alumno caracteriza el entorno del e-learning, parece claro que el aprendizaje autorregulado es un factor importante y crucial para su éxito. En este sentido, Dabbagh y Kitsantas (2012) sostienen que la formación en línea es un entorno educativo ideal para desarrollar habilidades de aprendizaje autorreguladas.

\section{AUTOMATIZACIÓN Y ANDAMIAJE EN LOS ENTORNOS VIRTUALES}

Los resultados descritos previamente indican que la posibilidad de desarrollo y promoción del aprendizaje autorregulado son significativos siempre que haya un 
buen diseño pedagógico del entorno. Por un lado, el e-learning exige autorregulación por parte del alumno y, por otro lado, precisa brindar apoyo externo al alumno (regulación externa) para evitar la sobrecarga cognitiva. Al ver todos estos desarrollos y objetivos, observamos que ayudar a los estudiantes a desarrollar habilidades avanzadas de autorregulación es uno de los mayores desafíos en el desarrollo de las tecnologías para el e-learning. Si partimos de la premisa de que los espacios de aprendizaje virtuales están formados por un amplio ecosistema, se hace necesario avanzar en la automatización de algunos procesos que proporcionen una rápida retroalimentación al estudiante. Las tendencias apuntan a diversas líneas de trabajo tales como el desarrollo de entornos personales de aprendizaje, el uso de los agentes inteligentes o tutores adaptativos y las analíticas de aprendizaje.

\section{Entornos personales de aprendizaje (PLE)}

Bartolomé y Steffens (2011) proponen una serie de criterios que los entornos de aprendizaje enriquecidos con tecnología deben cumplir con el fin de apoyar los procesos de autorregulación: a) alentar a los estudiantes a planificar su propia actividad de aprendizaje, b) proporcionar retroalimentación sobre el desempeño de las tareas con el fin de facilitar su seguimiento y la correcta autodirección del proceso de aprendizaje y c) proporcionar a los estudiantes los criterios para evaluar los resultados de su aprendizaje. En su análisis, estos autores identifican a los PLE como el mejor entorno capaz de cubrir los tres requisitos mencionados. Al mismo tiempo, distintos autores Yen, Tu, Sujo-Montes y Sealander (2016) llaman la atención sobre la necesidad de incorporar procesos de andamiaje en el propio PLE para facilitar la autorregulación del aprendizaje, dado que los aprendices pueden tener dificultades para formular sus objetivos, prever y planificar las tareas y estrategias más adecuadas para lograrlos, anticiparse al fracaso, etc.

Tal y como hemos mencionado previamente, los PLE buscan facilitar la personalización al permitir que cada persona utilice aquellas herramientas que considere más oportunas para su aprendizaje sin estar vinculadas a un entorno institucional concreto o a un período de tiempo específico. "Conforme nos acercamos a la visión ideal de las personas como aprendices emancipados que asumen el protagonismo y control de su propio aprendizaje es más y más complejo explicar cuáles son los rasgos clave de esa forma de entender la educación. Y ese es el núcleo central del enfoque PLE” (Adell y Castañeda, 2013, p. 46).

Existen diferentes aproximaciones para su materialización. Por ejemplo, en el proyecto Personal Learning Environments Reference Model realizado por la Universidad de Bolton en 2006, los investigadores identificaron 77 patrones diferentes de uso de aplicaciones que fueron clasificados en 8 categorías: 1) herramientas de chat y mensajería; 2) herramientas de trabajo en grupo; 3) herramientas de calendarización, programación y administración del tiempo; 4) herramientas de agregación de noticias; 5) weblogging y herramientas de publicación personal; 
6) software social; 7) herramientas de autoría y colaboración; 8) y herramientas de integración (Wilson, 2008). Idealmente, estas diversas aplicaciones deberían permitir la interoperatividad. No obstante, los desarrollos tecnológicos son todavía limitados por lo que el problema de este planteamiento es que todavía supone más una declaración de intenciones que una realidad.

\section{Agentes pedagógicos}

Otra aproximación interesante es el uso de tutores o agentes pedagógicos que también pueden ser muy adecuados en ambientes de aprendizaje híbridos y en línea, donde las actividades de los estudiantes pueden ser monitorizadas a través de programas y aplicaciones de seguimiento. Los agentes pedagógicos son tecnologías que permiten el seguimiento de las actividades del estudiante y que pueden adaptarse en tiempo real. El objetivo es proporcionar ayuda y feedback evaluando de forma constante los resultados obtenidos. El potencial de esta tecnología para la enseñanza y el aprendizaje sigue creciendo. En el terreno comercial, los desarrollos tecnológicos se centran en el aprendizaje adaptativo para reinventar sus servicios básicos de desarrollo de libros de texto y material didáctico. Por ejemplo, Pearson se ha asociado con Knewton para desarrollar MyLab \& Mastering ${ }^{2}$ que es una plataforma de aprendizaje adaptativo en matemáticas que se está aplicando en la Universidad de Arizona (Adams, Cummins, Davis et al., 2017).

Estos productos pueden combinarse con sistemas de recomendación que se definen como "elementos inteligentes de filtrado de información que proporcionan recomendaciones a la medida sobre productos destinados a un usuario" (Vazquez, 2015, p. 55). Tienen mecanismos para sugerir servicios, objetos o personas que son de interés en un contexto determinado lo cual implica que estos sistemas requieren filtrar o seleccionar información de los usuarios para, según ella, realizar recomendaciones. La interacción entre el usuario y el entorno virtual de aprendizaje incluye un conjunto de relaciones, en las que intervienen los componentes típicos de un sistema, por lo que se trata de un proceso dinámico e iterativo en el que de forma constante se obtiene información del estudiante, la cual se procesa y posteriormente se presentan recomendaciones.

Los sistemas de recomendación pueden proporcionar apoyo al usuario a través de sugerencias sobre las acciones a realizar en el curso, sugerir al participante la realización de actividades de aprendizaje, el seguimiento de rutas de navegación, la consulta de material educativo, contestar un mensaje en el foro, qué hacer ante determinado problema o tarea, o la sugerencia de cualquier otro recurso que permita potenciar y mejorar su proceso de aprendizaje (Santos y Boticario, 2012). Según esto, los participantes pueden recibir ayuda en el desempeño de las tareas del curso, con el propósito de (Vázquez, 2015, p. 1) mejorar el resultado de los procesos de aprendizaje facilitando los contenidos del curso más apropiados y las rutas de aprendizaje adaptadas a las necesidades de los estudiantes y b) promover 
la colaboración entre pares. Además, el sistema de recomendación puede sugerir al usuario nuevas acciones, considerando la información de sus preferencias personales o las de otros usuarios con características similares.

\section{Analíticas de aprendizaje}

Finalmente, consideramos que el uso de las analíticas de aprendizaje también puede ofrecer información relevante en el proceso de autorregulación del aprendizaje. Las analíticas de aprendizaje son reconocidas como una poderosa herramienta para ayudar a los estudiantes a reflexionar sobre su actividad de aprendizaje y, por lo tanto, obtener conocimiento sobre sus procesos de aprendizaje. Siemens y Baker (2012, p. 252) definen las analíticas de aprendizaje como "la medición, recopilación, análisis y presentación de datos sobre los estudiantes, sus contextos y las interacciones que se generan, para comprender el proceso de aprendizaje que se está desarrollando y optimizar los entornos en que se produce". Las analíticas apoyan la interpretación de un amplio espectro de datos producidas y recogidas sobre los estudiantes para orientar su producción académica, predecir acciones futuras e identificar elementos problemáticos (Gros, 2016). El objetivo de la recolección, registro, análisis y presentación de estos datos puede tener varias finalidades y destinatarios. No obstante, consideramos que las analíticas de aprendizaje deberían ser especialmente relevantes para los propios estudiantes, para fortalecer y dotar de autonomía progresiva el proceso de aprendizaje del alumnado. Para ello hay que determinar, por un lado, cuáles son los datos más representativos de los procesos de aprendizaje, para poderlas monitorizar adecuadamente. Por otra parte, hay que revisar qué tipo de acciones pueden derivarse de la interpretación de estos datos, rehuyendo mecanismos automatizados que recuerdan más a una enseñanza adaptativa que los procesos de aprendizaje en un marco conectivista y dejando la responsabilidad de la toma de decisiones sobre las ulteriores acciones a realizar en manos del propio estudiantado. Esto es especialmente importante ya que el autoconocimiento puede considerarse una habilidad metacognitiva clave para la autorregulación del aprendizaje. Por lo tanto, para utilizar realmente las analíticas para ayudar a los estudiantes a convertirse en aprendices autónomos, es necesario adoptar un enfoque centrado en el alumno.

\section{CONCLUSIONES}

En este artículo hemos descrito la evolución que ha tenido el e-learning destacando los importantes cambios acontecidos en los aspectos pedagógicos y tecnológicos. A pesar de la desconfianza inicial por esta modalidad formativa, el tiempo ha mostrado su eficacia y su uso se ha extendido y generalizado. La formación virtual ha estado muy ligada al uso de los gestores de contenidos educativos o LMS y estos se han 
implementado también en la enseñanza presencial. De todas formas, los LMS ya no son el único componente de la formación en línea. De hecho, el reto en el futuro es poder relacionar las diferentes herramientas y servicios disponibles para gestionar el aprendizaje.

Es importante investigar las peculiaridades del e-learning sin estar comparándolo constantemente con la formación presencial. De hecho, este tipo de formación precisa de un diseño pedagógico diferente a la enseñanza presencial y las investigaciones sobre el aprendizaje en línea destacan la importancia en este tipo de formación de la adquisición y gestión de competencias de autorregulación del aprendizaje.

Abrir los espacios más allá del aula virtual implica la diversificación de herramientas y aplicaciones digitales que precisan compartir datos e información de los aprendices. El uso de esta información puede permitir avanzar en la automatización de algunos procesos que proporcionen una rápida retroalimentación al estudiante. En esta línea, hemos señalado tres retos o tendencias: el desarrollo de entornos personales de aprendizaje, el uso de los agentes inteligentes y las analíticas de aprendizaje. Por tanto, consideramos que la sostenibilidad y el éxito futuro de la formación virtual deberá configurarse fusionando diferentes tipos de espacios y recursos mucho más específicos en función de las necesidades de los estudiantes.

\section{NOTAS}

1. Diferente terminología se ha utilizado para referirse al e-learning: aprendizaje en línea, enseñanza virtual, aprendizaje distribuido, formación virtual, enseñanza en línea, etc. En el texto los usamos indistintamente.

2. https://www.pearsonmylabandmastering.com/global/

\section{REFERENCIAS BIBLIOGRÁFICAS}

Adams, S., Cummins, M., Davis, A., Freeman, A., Hall Giesinger, C., y Ananthanarayanan, V. (2017). NMC horizon report: 2017 higher education edition. Austin, Texas: The New Media Consortium.

Adell, J., y Castañeda, L. (2013). El ecosistema pedagógico de los PLEs. En L. Castañeda y J. Adell (Eds.), Entornos Personales de Aprendizaje: Claves para el ecosistema educativo en red (29-51). Alcoy: Marfil.

Anderson, T., y Dron, J. (2011). Three generations of distance education pedagogy. The International Review of
Research in Open and Distance Learning, 12(3), 80-97.

Area, M., y Adell, J. (2009). eLearning: Enseñar y aprender en espacios virtuales. En J. De Pablos (Coord.), Tecnología Educativa. La formación del profesorado en la era de Internet (391-424). Málaga: Aljibe,

Bartolomé, A., y Steffens, K. (2011). Technologies for self-regulated learning. En R. Carneiro (Coord.), Self-Regulated Learning in Technology Enhanced Learning Environments (21-31). London: Sense Publishers. 
Cabero, J. (2013). El aprendizaje autorregulado como marco teórico para la aplicación educativa de las comunidades virtuales y los entornos personales de aprendizaje. Teoría de la Educación. Educación y Cultura en la Sociedad de la Información, 14(2), 133-156.

Chatti, MA., Jarke, M., y Specht, M. (2010). The ${ }_{3} \mathrm{P}$ Learning Model. Educational Technology y Society, 13(4), 74-85.

Cho, M. H., y Cho, Y. (2017). Self-regulation in three types of online interaction: a scale development. Distance Education, 38(1), 70-83.

Dabbagh, N., y Kitsantas, A. (2012). Personal Learning Environments, social media, and self-regulated learning: A natural formula for connecting formal and informal learning. The Internet and higher education, 15(1), 3-8.

Daniel, J., Vázquez Cano, E., y Gisbert, M. (2015). El futuro de los MOOC: ¿aprendizaje adaptativo o modelo de negocio? RUSC. Universities and Knowledge Society Journal, 12(1), 64-74. Recuperado de http://dx.doi. org/10.7238/rusc.v12i1.2475

Darabi, A., Liang, X., Suryavanshi, R., y Yurekli, H. (2013). Effectiveness of online discussion strategies: A meta-analysis. American Journal of Distance Education, 27(4), 228-241.

Dinçer, S., y Doğanay, A. (2017). The effects of multiple-pedagogical agents on learners' academic success, motivation, and cognitive load. Computers \& Education, 11(1), 74-100.

Downes, S. (2012). E-Learning generations. Recuperado de http://halfanhour. blogspot.be/2012/02/elearning generations.htm

García-Holgado, A., y García-Peñalvo, F. J. (2017). Gestión del conocimiento abierto mediante ecosistemas tecnológicos basados en soluciones Open Source. Congreso Ecosistemas del Conocimiento Abierto. Salamanca: Universidad de
Salamanca. doi: https://gredos.usal.es ispui/handle/10366/135570

García-Peñalvo, F. J., y Seoane-Pardo, A. M. (2015). Una revisión actualizada del concepto de eLearning. Décimo Aniversario. Education in the Knowledge Society, 16(1), 119-144. doi: http://dx.doi. org/10.14201/eks2015161119144

Gros, B., y García-Peñalvo, F. J. (2016). Future trends in the design strategies and technological affordances of e-learning. Learning, Design, and Technology: An International Compendium of Theory, Research, Practice, and Policy, 1-23.

Gros, B. (2016). Retos y tendencias sobre el futuro de la investigación acerca del aprendizaje con tecnologías digitales. Revista de Educación a Distancia, 32, 1-13.

Henderson, M., Selwyn, N., y Aston, R. (2017). What works and why? Student perceptions of 'useful'digital technology in university teaching and learning. Studies in Higher Education, 42(8), 1567-1579.

Kizilcec, R. F., Pérez-Sanagustín, M., y Maldonado, J. J. (2017). Self-regulated learning strategies predictlearnerbehavior and goal attainment in Massive Open Online Courses. Computers \& education, 104, 18-33. Recuperado de https://doi. org/10.1016/j.compedu.2016.10.001

Lang, C., Siemens, G., Wise, A., y Gasevic, D. (Eds.) (2017). Handbook of learning analytics. London: SOLAR. doi: http:/ dx.doi.org/10.18608/hla17

Lerís López, D., Vea Muniesa, F., y Velamazán Gimeno, A. (2015). Aprendizaje adaptativo en Moodle: Tres casos prácticos. Education in the Knowledge Society, 16(4), 138-157. doi: http://dx.doi. org/10.14201/eks201516138157

Nikolaki, E., Koutsouba, M., Lykesas, G., Venetsanou, F., y Savidou, D. (2017). The support and promotion of selfregulated learning in distance education. European Journal of Open, Distance and E-learning, 2O(1). 
Noesgaard, S. S., y Ørngreen, R. (2015). The effectiveness of e-learning: An explorative and integrative review of the definitions, methodologies and factors that promote e-learning effectiveness. Electronic Journal of e-Learning, 13(4), 278-290.

Rowe, F. A., y Rafferty, J. A. (2013). Instructional design interventions for supporting self-regulated learning: enhancing academic outcomes in postsecondary e-learning environments. Journal of Online Learning and Teaching, 9(4), 590-610.

Siemens, G., y d Baker, R. S. (2012). Learning analytics and educational data mining: towards communication and collaboration. En Proceedings of the 2nd international conference on learning analytics and knowledge (pp. 252-254). ACM. Recuperado de https://pdfs.semanticscholar.org/c245/a3403d6fd4064e6fd7666914a29afc7fe5ea.pdf

Siemens, G. (2005). Connectivism: Alearning theory for the digital age. International Journal of Instructional Technology and Distance Learning, 2(1), 3-10.

Siemens, G. (2014). Digital Learning Research Network. Learnspace, November. Recuperado de http://www. elearnspace.org/blog/2014/11/18/digitallearning-research-network-dlrn/

Siemens, G., Gašević, D., y Dawson, S. (2015). Preparing for the digital university: $A$ review of the history and current state of distance, blended, and online learning. Edmonton: Athabasca University.

Sung, Y. T., Chang, K. E., y Liu, T. C. (2016). The effects of integrating mobile devices with teaching and learning on students' learning performance: A meta-analysis and research synthesis. Computers \& Education, 94, 252-275.

Triquet, K., Peeters, J., y Lombaerts, K. (2017). Self-Regulated Learning Online:
Empirical Foundations, Promotion \& Evaluation for Teacher Professional Development. Contributing SRL Part to TeachUP. A policy experimentation cofunded by Erasmus+. Deliverable D1.1: Gaps in ITE and CPD provision report. Department of Educational Sciences, Brussels: Vrije Universiteit.

Van Laer, S., y Elen, J. (2017). In search of attributes that support self-regulation in blended learning environments. Education and Information Technologies, 22(4), 1395-1454.

Vázquez-Cano, E., Martín-Monje, E., y Castrillo de Larreta-Azelain, M. D. (2016). Analysis of PLEs' Implementation under OER Design as a Productive TeachingLearning Strategy in Higher Education. A Case Study at Universidad Nacional de Educación a Distancia. Digital Education Review, 29, 62-85.

Vázquez-Cano, E. (2015). El reto tecnológico para la sostenibilidad de los massive open online course (MOOC). Panorama, 9(17) 51-60.

Wilson, S. (2008). Patterns of Personal Learning Environments. Interactive Learning Environments, 16(1), 17-34.

Yen, C. J., Tu, C. H., Sujo-Montes, L., y Sealander, K. (2016). A Predictor for PLE Management: Impacts of Self-Regulated Online Learning on Students' Learning Skills. Journal of Educational Technology Development and Exchange (JETDE), 9(1), 3 .

Yoshida, H. (2016). Perceived Usefulness of "Flipped Learning" on instructional design for elementary and secondary education: with focus on pre-service teacher education. International Journal of Information and Education Technology, 6(6), 430-434. doi: 10.7763/ IJIET.2016.V6.727. 


\section{PERFIL ACADÉMICO Y PROFESIONAL DE LA AUTORA}

Begoña Gros Salvat. Doctora en Filosofía y Ciencias de la Educación por la Universidad de Barcelona. Coordinadora del Grupo de Investigación "Entornos y materiales para el aprendizaje”. Miembro del consejo de dirección del Instituto de Investigación en Educación de la Universidad de Barcelona. Responsable de numerosas investigaciones nacionales e internacionales y de la dirección de tesis doctorales. Ha sido Vicerrectora de innovación e investigación en la Universitat Oberta de Catalunya (2007-11). Ha publicado diversos libros y artículos sobre las tecnologías digitales para el aprendizaje. Sus principales líneas de investigación se centran en la aplicación de las tecnologías digitales para el aprendizaje y la innovación educativa.

E-mail: bgros@ub.edu

\section{DIRECCIÓN DE LA AUTORA}

Facultad de Educación

Passeig Vall d'Hebron, 171

o8035 Barcelona

Fecha de recepción del artículo: 24/11/2017

Fecha de aceptación del artículo: 18/12/2017

\section{Como citar este artículo:}

Gros Salvat, B. (2018). La evolución del e-learning: del aula virtual a la red. RIED. Revista Iberoamericana de Educación a Distancia, 21(2), pp. 69-82. doi: http://dx.doi.org/10.5944/ried.21.2.20577 